\title{
Pop-Culture Learning Technique Applied to Thermodynamics
}

\section{Dr. Laura A. Garrison, York College of Pennsylvania}

Dr. Laura Garrison received her B.S. in Mechanical Engineering from the University of Texas and her M.S. in Operations Research from Stanford University. She then worked for AT\&T Bell Laboratories and AT\&T Federal Systems before deciding to pursue her Ph.D. in Bioengineering at Penn State University in the area of experimental fluid mechanics associated with the artificial heart. After graduating, she worked at Voith Hydro for five years in the area of Computational Fluid Mechanics. For the last fifteen years, she has been a professor at York College of Pennsylvania where she teaches thermal sciences, freshmen design courses, and computer programming. 


\section{Pop-Culture Learning Technique Applied to Thermodynamics}

A recent TEDx talk by Josh Kaufman claims that almost anything can be learned in 20 hours. ${ }^{1}$ One of the key recommendations is to break the skill into its most basic elements and master these small skills before moving on. Mr. Kaufman claims that this technique can be applied to any topic, from playing the ukulele to learning computer programming. By studying people who perform at the top of their field, other researchers have also come to the conclusion that this technique works well.

Many students struggle with thermodynamics, in part because the individual problems tend to be very comprehensive, requiring many skills. To apply Kaufman's philosophy to thermodynamics, the author developed a large number of on-line Moodle "quizlets" that provide students with repetitive practice of the most basic skills in the course. For example, there is an entire quizlet based on finding the phase of a substance using tables. If the students don't get a "mastery level” score, they take a subsequent quiz on phases before moving on. Surveys and grades suggest that there were substantial improvements in student learning using these quizlets. Because of this success, the author is currently developing new software that could potentially be used by any instructor to incorporate these small skills into their thermodynamics course. The software will incorporate the quizlet questions as well as techniques used by video game developers.

The paper presents more details on the quizlet questions and assessment. It also describes the current state of the new software and how it is being incorporated into a thermodynamics course.

\section{Introduction}

Recent research shows that the traditional lecture-based course structure is not the most effective teaching method. ${ }^{2}$ Many instructors have been adding active learning to the classroom, with the "flipped" classroom becoming very popular. ${ }^{3}$ The author has experimented with flipping classes with mixed results, finally landing on a "half-flipped" structure with short lectures followed by problem-solving. The question then becomes: How do you make the most of the active learning period? Answering individual questions while students work on homework problems wasn't as effective as the author would like. There were issues with both group and individual work and the students sometimes had trouble staying on task. In a TedX talk and in a book, Josh Kaufman claims that anything can be learned in 20 hours by breaking the activity down into its most essential small skills and repeating those skills until they are mastered before moving on. ${ }^{1,4}$ In Outliers, The Story of Success, Malcolm Gladwell claimed that approximately 10,000 hours of practice is necessary to develop world-class talent, and those that reach this level do not necessarily have any special genetic advantage. ${ }^{5}$ While college students do not need to become world-class talents, and 20 hours of practice is probably not enough, the techniques that are used to develop these talents can be used to make the learning process more efficient in an academic setting. In The Talent Code: Greatness isn't Born. It's Grown. Here's How and in The Little Book of Talent, Daniel Coyle claims that talent comes from growing myelin on nervous system pathways and the most efficient way to do this, as Kaufman also claims, is to break down 
the talent into small "clusters" of skills, work at the edge of one's ability, and repeat the skills many times, with each success building more myelin on the associated nerves. ${ }^{6,7}$ This myelin allows us to learn so well that we can do many tasks without thinking at all.

Because Thermodynamics problems tend to be long and comprehensive, the author decided that it might be beneficial to break out the necessary simple skills and have the students repeat them to a mastery level before attempting the bigger problems. To incorporate small-skill practice into the class, the author developed a series of on-line Moodle "quizlets" and moved the course to a computer lab so these quizlets could be completed during the class period. Over 300 problems were developed and entered into Moodle. The quizlets were used in two sections of approximately 23 students each in the spring of 2015 and approximately the same number in the Spring of 2016. The exam problem format did not change from previous semesters and homework and other larger practice problems were still distributed. Thus, the students still needed to complete the large problems in order to pass the exams. Student surveys and grades were used to assess the effectiveness of the quizlets.

\section{Quizlet Description}

Before attempting each of the 52 quizlets, the students were given practice problems via a worksheet. An example worksheet problem is shown in figure 1 . Note that the goal of the example practice problem was to achieve proficiency with unit conversions. Figure 2 is a screen snapshot showing the Moodle interface for the first few quizlets. As indicated in figure 2, the students who achieved a target grade would receive bonus points on their daily notes quiz. There was an intention to give the students a chance to take a second quizlet if they didn't reach the target grade on the first attempt. However, the instructor quickly ran out of time and the retakes stopped within the first two weeks of class.

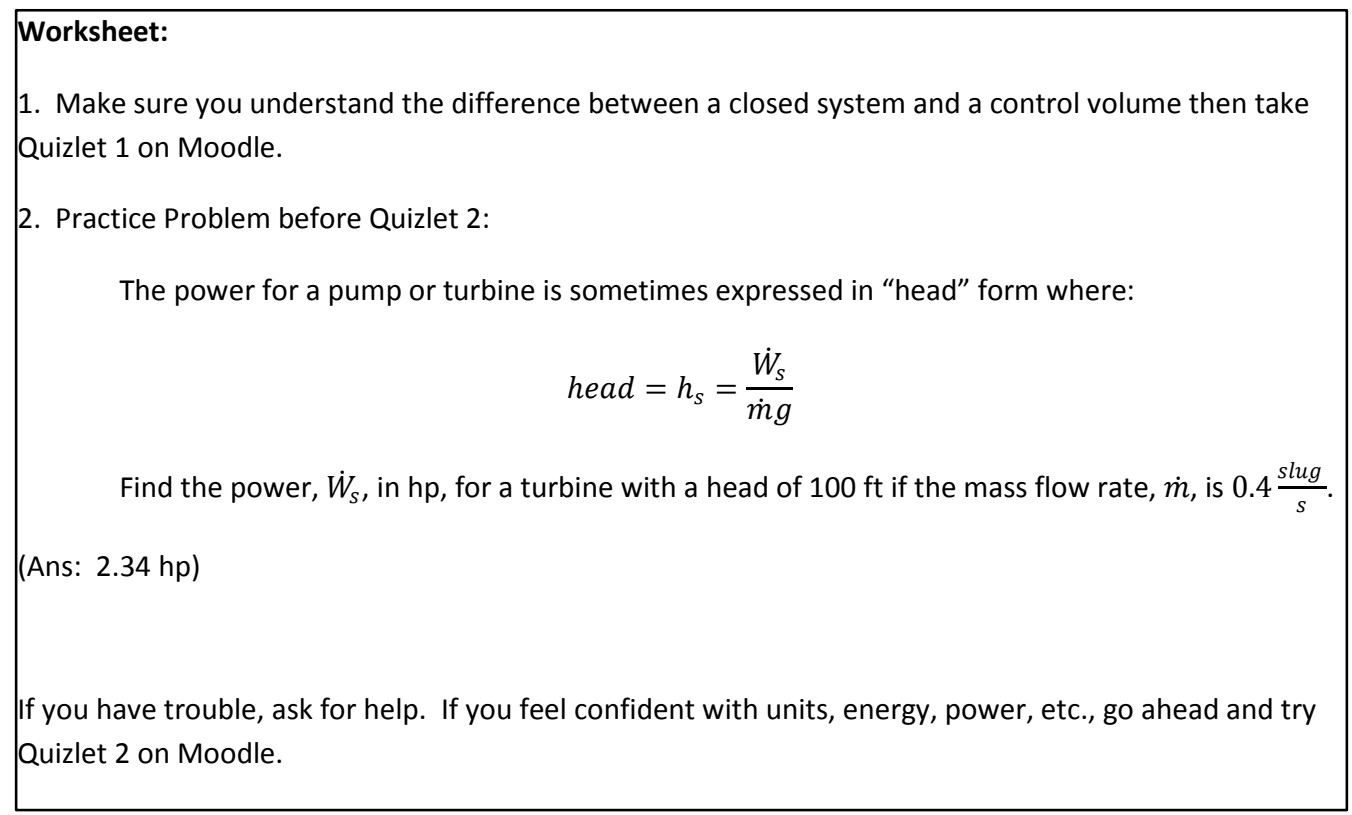

Figure 1: An example worksheet 


\section{Quizlets}

Unless otherwise specified, these quizlets are open book, open notes.

\section{Quizlet 1 - Closed Systems vs Control Volumes}

Get 4 out of 5 correct for 1 bonus point on your Notes Quiz. If you don't know what the object in a question does or looks like, please ask before trying to answer the question.

If you don't get 4 out of 5 correct, take the Quizlet 1 Retake for a chance to get 0.5 bonus points on your Notes Quiz.

Quizlet 1 Retake - Closed Systems vs Control Volumes

Everyone is welcome to take this quizlet, but you only get credit for it if you did not get 4 out of 5 correct on the original Quizlet. You need 4 out of 5 correct to receive 0.5 bonus points on your Notes Quiz. If you don't get 4 out of 5 correct, ask the instructor to help figure out what you are doing wrong

Quizlet 2 - Units

Get 3 out of 4 problems correct for 1 bonus point on your Notes Quiz. Enter numerical answers without units.

Quizlet 2 Retake - Units

If you didn't get the point for the problems on the original, take this quizlet. Get 3 out of 4 correct for 0.5 bonus point on your Notes Quiz.

Figure 2: Students select the quizlets for the day from the above interface.

Example questions from a variety of topics are shown below. The intent was to break the larger topics down to basic skills and have the students repeat these skills until proficient. There were typically 6-10 questions per quizlet.

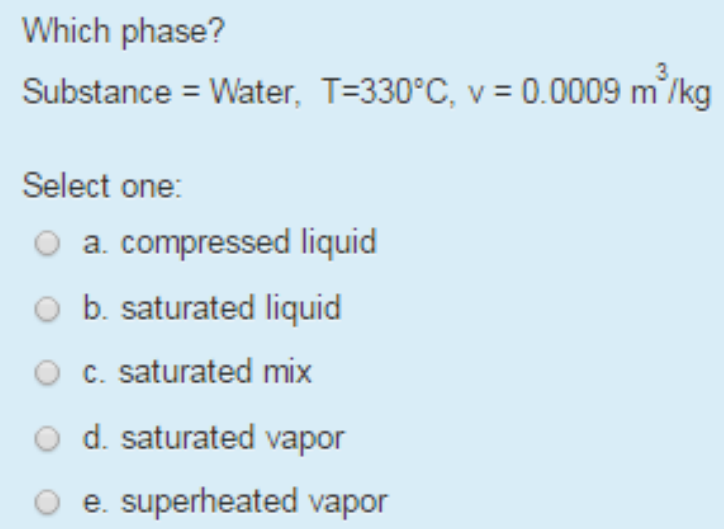


Substance: water

$\mathrm{T}=180^{\circ} \mathrm{C}, \mathrm{v}=0.001107 \mathrm{~m}^{3} / \mathrm{kg}$

Find $p$ in MPa. Include 4 significant digits and be careful with rounding.

Answer:

\section{CHECK}

A system that contains the entire universe is a

Select one:

a. closed system

b. control volume

CHECK

The inside of your freezer at home constitutes which type of thermal reservoir?

Select one:

a. $T_{L}$

b. $T_{H}$

c. $\mathrm{Bi}$

d. Neither $T_{H}$ nor $T_{L}$

\section{CHECK}

Air enters an insulated, reversible turbine at $1040 \mathrm{~K}, 277.7 \mathrm{kPa}$ and exits at $120 \mathrm{kPa}$. Find the enthalpy at the exit in $\mathrm{kJ} / \mathrm{kg}$ using variable specific heats. $3 \mathrm{sig}$. figs.

Answer:

\section{CHECK}




\section{Comparison Between Quizlets and Textbook Problems}

Prior to 2015, the author used traditional homework assignments mainly taken from the Cengel and Boles textbook to provide students with practice problems. ${ }^{9}$ A total of 76 problems were assigned. In comparison, 331 quizlet problems were assigned, some from textbooks, but a majority derived from scratch. The reason that the students can complete many more quizlet problems than publisher-provided problems is because the quizlet problems start out simple and ramp up to more complex, comprehensive problems. The philosophy behind most of the textbook problems is to challenge the students by applying multiple topics into real-world-type scenarios. There are typically a few simple problems in the textbook on a topic, but the majority are more complex. It is vital that students learn to do these types of challenging problems, but it is difficult for them to jump straight from lecture to these more complex applications. The quizlets provide the students with much more repetition on the new topics before asking them to attempt the more challenging homework-type problems. This section illustrates differences between the available publisher's problems from the Cengel and Boles textbook and the quizlet problems by comparing the questions for one week in the middle of the semester. ${ }^{9}$ The topics were entropy and isentropic (constant entropy) processes.

In the years before quizlets were introduced, practice for this week consisted of a homework assignment containing one concept question and eleven problems from the textbook. In addition, since the class was partially flipped, there were two in-class worksheets. The worksheets contained a total of three large challenging problems. Below is one of the assigned homework problems. The solution from the textbook is given in Appendix A. ${ }^{9}$

7-77 An insulated piston-cylinder device initially contains $300 \mathrm{~L}$ of air at $120 \mathrm{kPa}$ and $17^{\circ} \mathrm{C}$. Air is now heated for 15 minutes by a $200-\mathrm{W}$ resistance heater placed inside the cylinder. The pressure of air is maintained constant during this process. Determine the entropy change of air, assuming (a) constant specific heats and (b) variable specific heats. ${ }^{9}$

By thermodynamics standards, this is a short problem. Many textbook problems have multiplepage solutions. Though relatively short, students still needed a significant amount of time to remember the first law and ideal gas law concepts required to figure out the solution. For this reason, only a small number of problems could be assigned at one time. Thus practice on the new topics was somewhat limited.

After quizlets were introduced, practice for this week consisted of in-class worksheet problems with answers followed by quizlets on the same topics. Many of the quizlet problems are completed in class. The same homework assignments as previous semesters were provided, but were optional. The required work for the same week as the above referenced homework is summarized here and samples are provided in Appendix B:

Day 1:

Quizlet 1: Ten multiple choice questions on isentropic processes (constant entropy) 
Worksheet Problem: A practice problem similar to those found in Quizlet 2 (described below) but broken down into five steps.

Quizlet 2: Five work-out problems on isentropic devices involving steam or R134a. Note that it is easier to find entropy for steam and R-134a than for gases.

Day 2:

Worksheet Problem: A two-part problem, each part similar to problems found in Quizlet 1.

Quizlet 1: Ten short problems on finding entropy changes for ideal gases and incompressible substances.

Worksheet Problem: A problem similar to Quizlet 2 problems.

Quizlet 2: Four comprehensive problems on using entropy. These were more like homework problems.

Day 3:

Quizlet 1: Five problems on isentropic processes involving air. These build to the larger Otto cycle problems.

Quizlet 2: A seven-part problem on the air-standard Otto cycle. Each part was a separate quizlet problem. This problem was from the Moran and Shapiro textbook. ${ }^{8}$

Quizlet 3: A five-part problem repeating the previous quizlet but using cold-air-standard analysis. ${ }^{8}$

With the new structure, the problems start out simple and repetitive but then build up to the larger problems. This learning process was efficient enough that the instructor was able to cover extra material in that week, thus the introduction of the Otto cycle (Day 3, Quizlets 2 and 3).

\section{Assessment}

In 2015 and 2016, surveys were distributed approximately one month into the semester. These surveys included the free-response question: "What aspect(s) of the class help you the most and why?” Results from that question are summarized in Figure 3. 

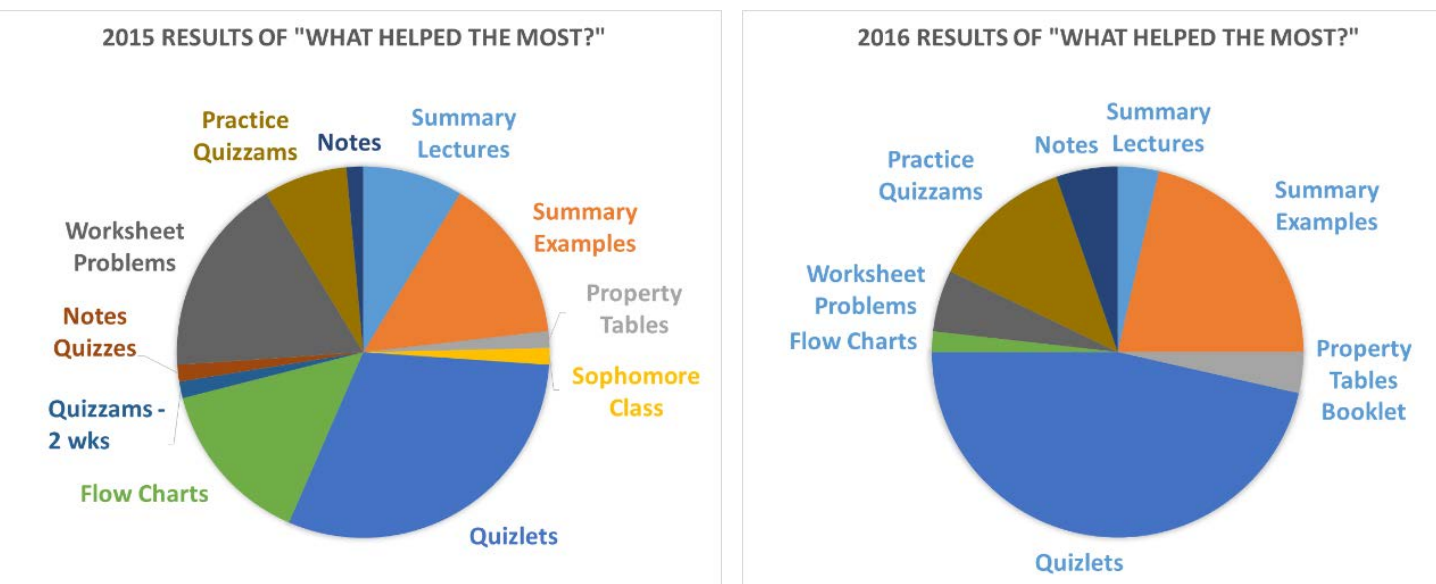

Figure 3: Summarized results of the survey question "What aspect(s) of the class help you the most and why?” given approximately one month into the semester in 2015 (left) and 2016 (right). Quizlets are in a medium blue.
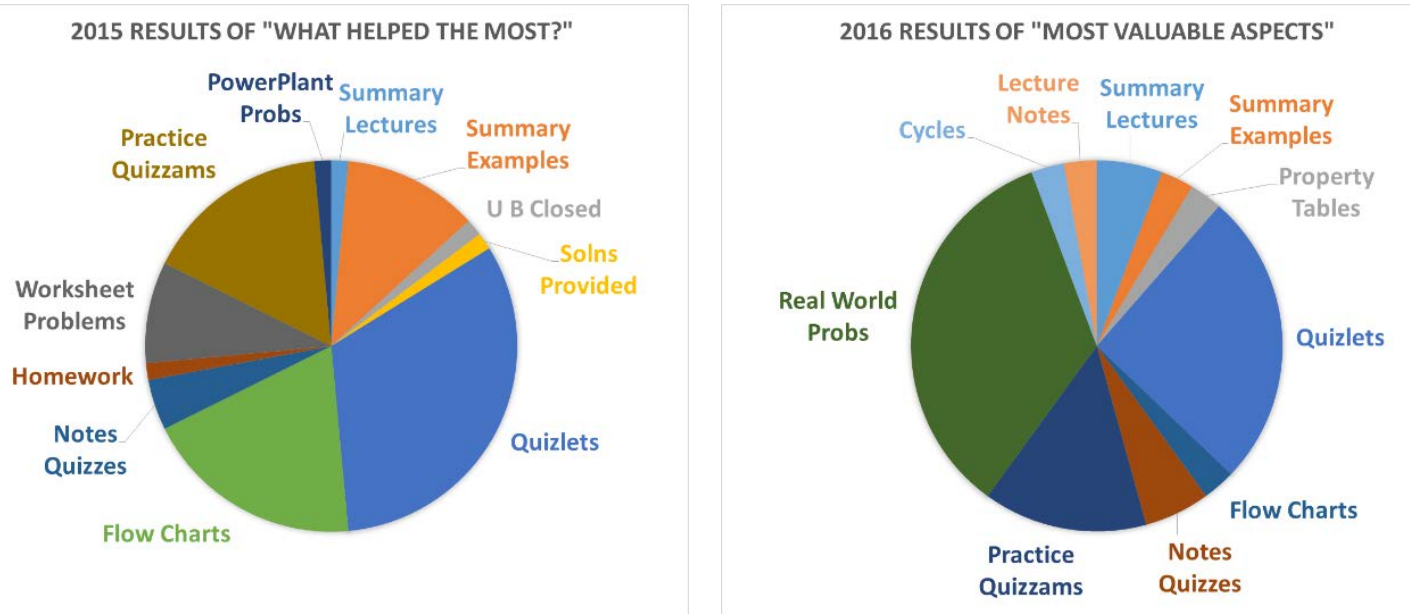

Figure 4: Summarized results of the survey question "What aspect(s) of the class help you the most and why?" given at the end of the semester in 2015 (left) and results of the question "Which aspects of this course were most valuable?" given at the end of the semester in 2016 (right). Quizlets are in a medium blue.

Both years, students cited quizlets more than any other aspect of the course as being most helpful. The quizlet content did not change significantly between 2015 and 2016, but the students in 2016 seemed to think the quizlets were more helpful. In 2015, the same survey was given after the final exam and is summarized in Figure 4. The instructor did not administer the survey at the end of the semester in 2016 because the final was optional that year. However, a similar question in the college-administered course evaluation reads: "Which aspects of this course were most valuable?" Results of that question are summarized in Figure 4. At the end of the semester, the students still ranked quizlets as the most helpful aspect of the course in 2015, and second most valuable aspects in 2016 after "Real World Problems," which is more about course content than learning. Although the student opinions 
are an indirect measure of effectiveness, they do a good job of targeting just the quizlets. It is significant that so many of the students, without any prompting or multiple choice selections, ranked quizlets as the aspect of the course that helped them the most. The author believes that student surveys can be powerful assessment tools, even if indirect.

While students indicated that quizlets were very helpful, a more direct assessment was the final exam which was the same in 2014 (before quizlets) and 2015 (after quizlets were introduced). The final exam average jumped from $63 \%$ in 2014 to $74 \%$ in 2015 . At the time, this jump in grades seemed astounding. However, the group of students that took thermodynamics in 2015 turned out to be exceptional. The instructor had access to the mechanical engineering students' transcripts and, neglecting the students who were repeating the course or who received and F, their average GPAs jumped from 3.14 in 2014 to 3.47 in 2015. Also, other changes in the course such as changing the number of exams and adding a "mastery" requirement to finding properties make it difficult to separate the effect of the quizlets from the other changes. Still, the author believes that the quizlets were a big part of the grade improvements. Note that the final exam was optional in 2016, so only the students with the lowest averages took it, and it cannot be compared to the previous years.

The following list includes some other observations regarding the quizlets. Many of these indicate the benefits of the on-line aspect for which students receive instant feedback.

- Students really hate getting the answer wrong in Moodle quizzes (they receive a pink box instead of green).

- Double- and triple-checking their answers became commonplace.

- Students were more likely to go back and check their units.

- They became better at determining which digits are significant.

- Instant feedback is very much appreciated.

- Even though students seem to understand the topics during a lecture or while doing examples, many times they can't get started on even the simple problems. The same concepts must be repeated many times before they actually sink in and they usually have to make the same mistake several times before they really get it. Watching the students attempt the quizlets was very enlightening.

- Later in the semester, the promise of bonus points became less of a motivator and the quizlet completion rate dropped off.

- Incorrect answers are pink on the student's computer screen, so it is easy to see when students are having trouble. By asking what went wrong, the instructor can force the student to review their work. This allows for more student-instructor interactions that specifically target problem areas.

\section{Current and Future Plans}

The author is currently using the quizlets in Spring of 2017 and intends to continue using and enhancing them for the future. Changes to the course include:

- Instead of bonus points, quizlets are worth $7 \%$ of the overall grade. 
- A journal requirement was added and the students do their quizlet work in their journal.

- On the first day of class, the instructor played a portion of the Kaufman TedX talk in class, which helped to explain the reasons behind the structure of the course to the students. ${ }^{1}$

- The order of topics was reorganized (see Section 4.1)

- New software was developed to administer the quizlets and enhance the student experience. (see Section 4.2)

\subsection{Reorganization}

Much of the real-world applications of thermodynamics occurs in the sections on cycles. Typically, textbooks cover almost all of the background information needed for all of the cycles before starting on any cycle. It is typically chapters 8 - 11 before analysis of vapor or gas cycles are introduced. ${ }^{8,9,10}$ The author believes that students have less enthusiasm for the material than if they were clearly working toward the analysis of a cycle from the beginning. For the current semester, the course has been reorganized. Power plants are introduced on the first day. Students are taught solely what they need to know for the Rankine cycle, including finding properties from steam tables, first and second laws for control volumes, and cycle analysis. The students analyze basic Rankine cycles by the fifth week of class. After finishing Rankine cycles with reheat and regeneration, topics necessary to analyze jet engines (gas turbine power plants) will be introduced. This will include finding properties for gases along with first and second laws for nozzles and diffusers. Following jet engines, Otto and Diesel cycles will be covered along with the first and second laws for closed systems. Finally, mixtures and psychrometrics will be covered so the students can analyze refrigeration cycles and heating and cooling ducts.

\subsection{Software}

Although Moodle provided a nice quiz interface, the instructor desired some features that Moodle did not have. During a sabbatical, the author developed a web application to administer the quizlets. This software, called Unlocking Thermodynamics, incorporates techniques utilized by video game developers to keep people playing. ${ }^{11,12}$ Some of the current features include:

- Graphic representation of progress through the cycles.

- Target grades with additional problems if a target is not attained.

- Problems in random order but categorized by easy, medium, hard with easy problems first.

- Bonus points given at random. Fireworks animation when a bonus point is achieved. These random bonus points are weighted to occur more towards the beginning of the semester.

- Only one question at a time can be skipped, so a student cannot necessarily work on the same problem as a neighboring student.

- High scores by section.

- Continuous grade display while taking the quizlet. 
The beta version of this software is currently being used in the spring 2017 semester and is working well. Figures 5 and 6 are screenshots. The software has been designed to be shared with other instructors but it still needs some work before this can happen. Unlocking Thermodynamics will be demonstrated at the conference.
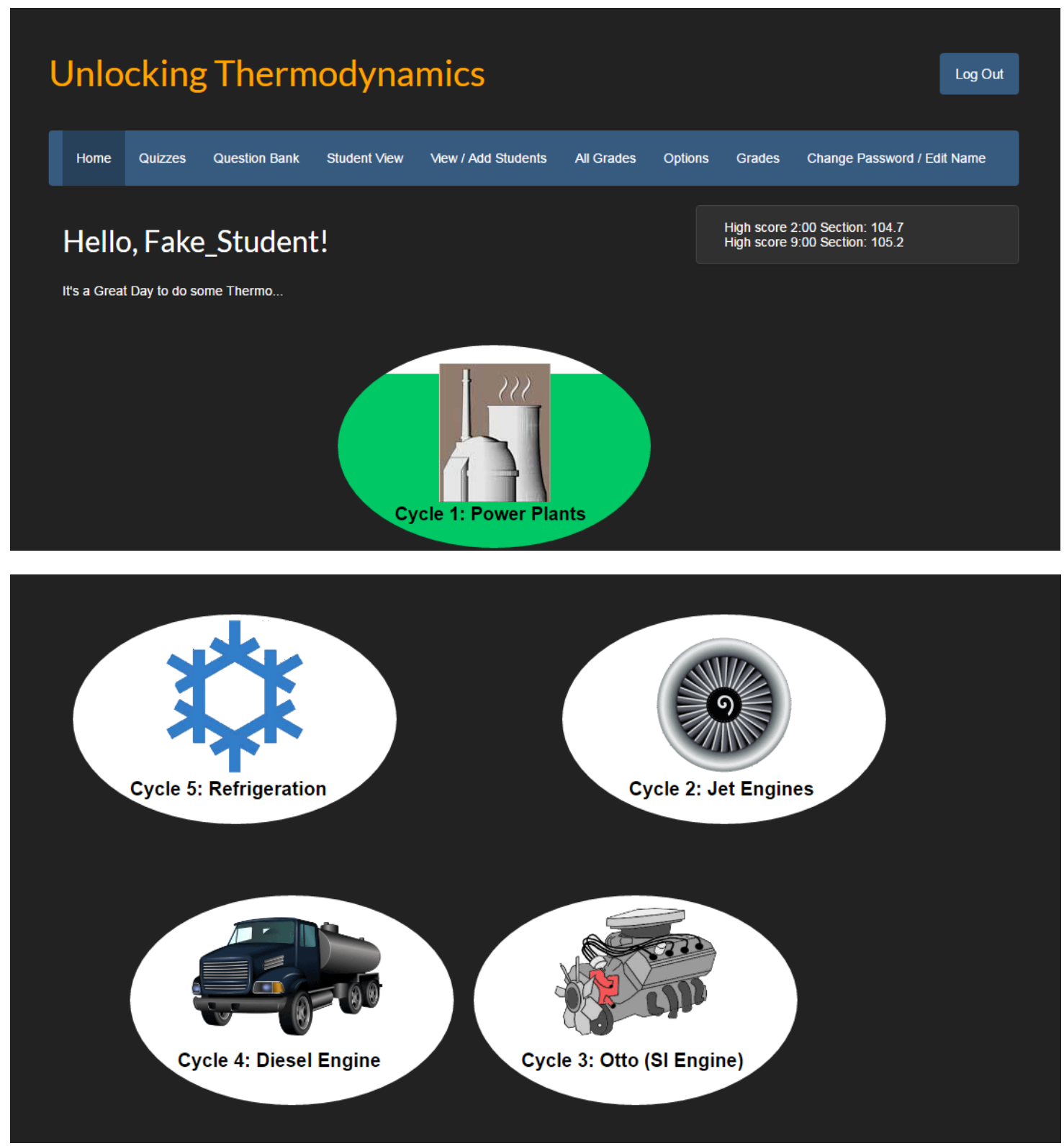

Figure 5: Two screenshots showing the top and bottom of the Home page of Unlocking Thermodynamics. This is an instructor's view. Students have just the Home, Grades, and Change Password options on the navigation bar. To select quizlets, the students click on the oval of the cycle they are working on. The green in the first oval indicates progress through that cycle. The high scores for each section are noted in the upper right. 


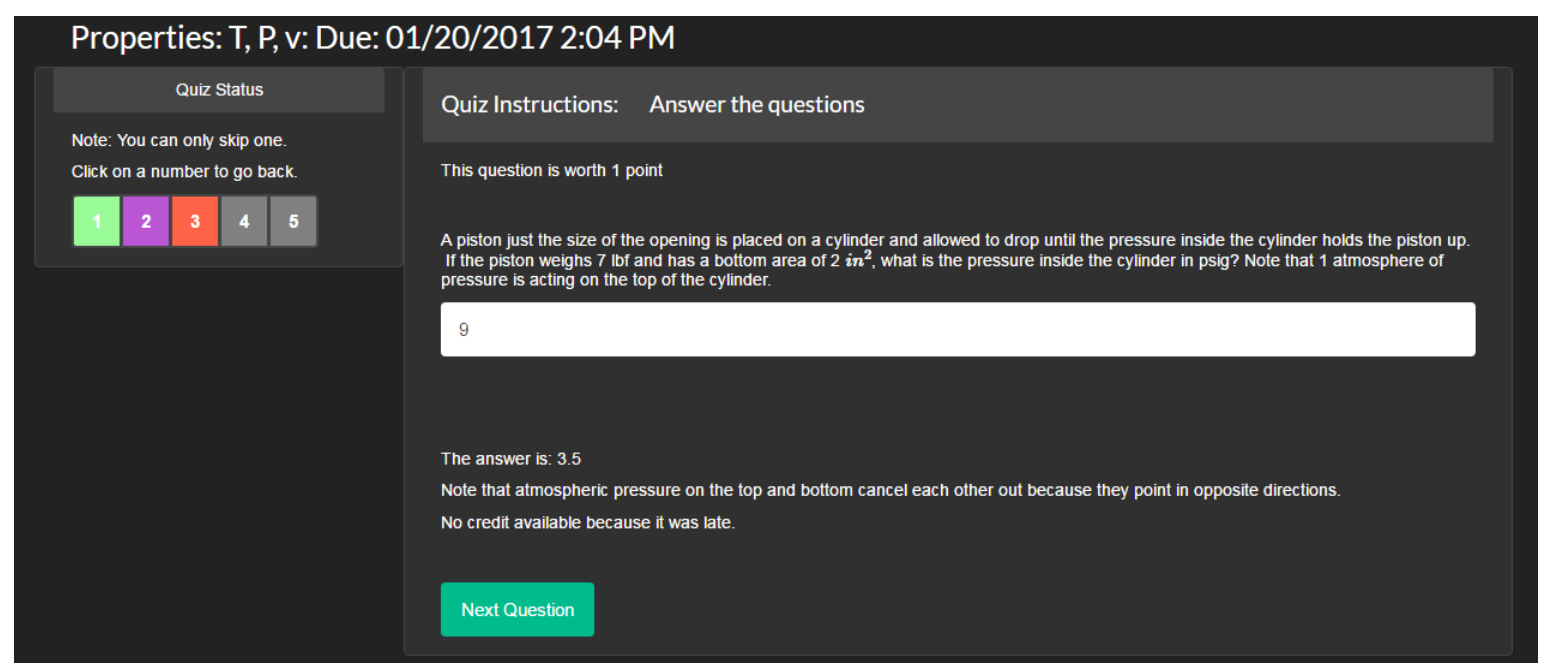

Target Grade: 80\%

Your Current Grade: $50.00 \%$

You have a $100 \%$ deduction on this question for being late

Figure 6: Two screen shots showing the top and bottom of a question screen. The green box indicates the student got the first question correct, the purple indicates a correct answer with bonus, and the red designates an incorrect or late answer. The boxes can also be yellow, indicating partial credit for being one day late or for needing more than one attempt on the question.

\section{Conclusions}

Quizlet problems were developed in an attempt to improve learning by breaking the topics into small portions and providing repetition. In two semesters of implementation, the students indicated that the quizlets were the most helpful aspect of the course and grades seemed to improve. Because the quizlets were administered mostly in class via computer software, the students not only benefitted from the content and repetition, but also from instant feedback, increased instructor interaction, and psychological factors such as an increase in effort due to their dislike of pink boxes for incorrect answers.

Once enough functionality is added to Unlocking Thermodynamics the author intends to make it available to other instructors. 


\section{References}

1. Kaufman, J "The first 20 hours - how to learn anything," [https://www.youtube.com/watch?v=5MgBikgcWnY Retrieved: Jan. 2017.

2. Prince, M. "Does Active Learning Work? A Review of the Research.” Journal of Engineering Education 93(3) 223-231, 2004.

3. Mason, G., \& Shuman, T. R., \& Cook, K. E. (2013, June), Inverting (Flipping) Classrooms Advantages and ChallengesPaper presented at 2013 ASEE Annual Conference, Atlanta, Georgia. https://peer.asee.org/19842

4. Kaufman, J., The First 20 Hours, How to Learn Anything Fast, New York, New York: Penguin Group, 2013

5. Gladwell M. Outliers, The Story of Success, New York: Little, Brown and Company, 2008

6. Coyle, D. The Talent Code, New York, New York: Bantam Dell, A Division of Random House, Inc, 2009

7. Coyle, D. The Little Book of Talent, New York, New York: Bantam Dell, A Division of Random House, Inc, 2012

8. Moran, M.J., Shapiro, H.N. Fundamentals of Engineering Thermodynamics, Hoboken, N.J.: John Wiley and Sons, Inc., $7^{\text {th }}$ ed., 2008.

9. Cengel, Y.A., Boles, M.A., Thermodynamics, An Engineering Approach, New York: McGraw Hill, $8^{\text {th }}$ ed., 2015

10 Borgnakke, C, Sonntag, R.E., Fundamentals of Thermodynamics, Hoboken, N.J.: John Wiley and Sons, Inc., $7^{\text {th }}$ ed., 2008.

11. Cundy, M., “Top 7...tricks that make video games highly addictive” [http://www.gamesradar.com/top-7-tricks-that-make-video-games-highly-addictive, Retrieved: Jan. 2017.

12. Wong, D. “5 Creepy Ways Video Games are Trying to Get You Addicted”, [http://www.cracked.com/article_18461_5-creepy-ways-video-games-are-trying-to-get-youaddicted.html, Retrieved: Jan. 2017. 


\section{Appendix A: Solution to a typical homework problem on the topic of entropy. ${ }^{9}$}

7-77 An insulated cylinder initially contains air at a specified state. A resistance heater inside the cylinder is turned on, and air is heated for $15 \mathrm{~min}$ at constant pressure. The entropy change of air during this process is to be determined for the cases of constant and variable specific heats.

Assumptions At specified conditions, air can be treated as an ideal gas.

Properties The gas constant of air is $R=0.287 \mathrm{~kJ} / \mathrm{kgK}$ (Table A-1).

Analysis The mass of the air and the electrical work done during this process are

$$
\begin{aligned}
& m=\frac{R U_{1}}{R T_{1}}=\frac{(120 \mathrm{kPa})\left(0.3 \mathrm{~m}^{3}\right)}{\left(0.287 \mathrm{kPa} \cdot \mathrm{m}^{3} / \mathrm{kg} \cdot \mathrm{K}\right)(290 \mathrm{~K})}=0.4325 \mathrm{~kg} \\
& W_{\text {o,in }}=W_{\text {o.in }} \Delta t=(0.2 \mathrm{~kJ} / \mathrm{s})(15 \times 60 \mathrm{~s})=180 \mathrm{~kJ}
\end{aligned}
$$

The energy balance for this stationary closed system can be expressed as

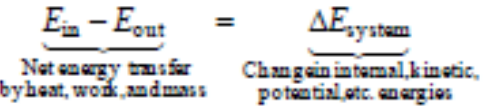

$$
\begin{aligned}
& W_{\text {a.in }}-W_{\text {b out }}=\Delta U \longrightarrow W_{\text {ain }}=m\left(h_{2}-h_{1}\right) \cong c_{p}\left(T_{2}-T_{1}\right)
\end{aligned}
$$

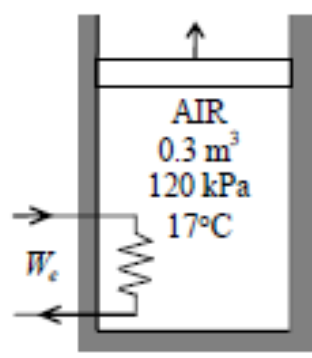

since $\Delta U+W_{\mathrm{b}}=\Delta H$ during a constant pressure quasi-equilibrium process.

(a) Using a constant $c_{p}$ value at the anticipated average temperature of $450 \mathrm{~K}$, the final temperature becomes Thus,

$$
T_{2}=T_{1}+\frac{W_{\mathrm{s}, \mathrm{m}}}{m c_{p}}=290 \mathrm{~K}+\frac{180 \mathrm{~kJ}}{(0.4325 \mathrm{~kg})(1.02 \mathrm{~kJ} / \mathrm{kg} \cdot \mathrm{K})}=698 \mathrm{~K}
$$

Then the entropy change becomes

$$
\begin{aligned}
\Delta S_{\mathrm{sys}} & =m\left(s_{2}-s_{1}\right)=m\left(c_{p, \mathrm{svg}} \ln \frac{T_{2}}{T_{1}}-R \ln \frac{P_{2}{ }^{90}}{R}\right)=m c_{p, \mathrm{xvg}} \ln \frac{T_{2}}{T_{1}} \\
& =(0.4325 \mathrm{~kg})(1.020 \mathrm{~kJ} / \mathrm{kg} \cdot \mathrm{K}) \ln \left(\frac{698 \mathrm{~K}}{290 \mathrm{~K}}\right)=0.387 \mathrm{~kJ} / \mathrm{K}
\end{aligned}
$$

(b) Assuming variable specific heats,

$$
W_{\text {o.in }}=m\left(h_{2}-h_{1}\right) \longrightarrow h_{2}=h_{1}+\frac{W_{\text {ain }}}{m}=290.16 \mathrm{~kJ} / \mathrm{kg}+\frac{180 \mathrm{~kJ}}{0.4325 \mathrm{~kg}}=706.34 \mathrm{~kJ} / \mathrm{kg}
$$

From the air table (Table A-17, we read $s_{2}^{\circ}=2.5628 \mathrm{~kJ} / \mathrm{kg} \cdot \mathrm{K}$ corresponding to this $h_{2}$ value. Then,

$$
\Delta S_{\mathrm{sys}}=m\left(s_{2}^{\circ}-s_{1}^{\circ}+R \ln \frac{P_{2}{ }^{\circ 0}}{R}\right)=m\left(s_{2}^{\circ}-s_{1}^{\circ}\right)=(0.4325 \mathrm{~kg})(2.5628-1.66802) \mathrm{kJ} / \mathrm{kg} \cdot \mathrm{K}=0.387 \mathrm{~kJ} / \mathrm{K}
$$




\section{Appendix B: Sample quizlet problems from the week covering entropy}

Day 1, Quizlet 1, three of the ten multiple-choice questions

Air in a piston/cylinder goes through an ideal expansion process for which $T_{1}=T_{2}$. This process is

Select one:

a. isentropic but not isothermal

b. isothermal but not isentropic

c. isothermal and isentropic

d. isentropic and isenthalpic

\section{CHECK}

For an isentropic turbine, the specifc form of the first law reduces to

Select one:

a. $q-w=\Delta u$

b. $w=u_{1}-u_{2}$

c. $w=h_{1}-h_{2}$

d. $q=\Delta u$

e. $q-w=h_{2}-h_{1}$

\section{CHECK}

If a compressor in a Carnot (ideal) refrigeration cycle has no heat transfer,

Select one:

a. the second law of thermodynamics does not apply

b. $s_{2}=s_{1}$

c. the first law of thermodynamics does not apply

d. $s_{2} \neq s_{1}$

e. you can't tell if the process is isentropic or not

\section{CHECK}




\section{Day 1: Worksheet problem}

1. Steam enters a throttle at $6 \mathrm{MPa}, 540^{\circ} \mathrm{C}$ and exits the throttle at $4 \mathrm{MPa}$ before entering a turbine. The steam then expands adiabatically through a turbine to $100 \mathrm{kPa}$. Determine the maximum power that could be generated by the turbine per kg of steam flowing through it.

(Ans: $910 \mathrm{~kJ} / \mathrm{kg}$ )

Step 1: Draw a schematic, labeling inlets and exits for each device. You should have states 1, 2, and 3:

Step 2: Write and simplify the first law for the turbine.

Step 3: Analyze the throttle, so that you have two properties at state 2:

Step 4: For maximum power, what do you know about entropy across the turbine? Use this fact to find two properties at state 3 . Then use this info to find the property you need to solve the equation from step 2.

Step 5: Solve the equation from step 2 to get your answer:

\section{Day 1: Three of 5 problems in Quizlet 2.}

A steam turbine, assumed to be $100 \%$ efficient (i.e. reversible) and adiabatic, has an inlet at 100 psia and $400^{\circ} \mathrm{F}$. If the exit is at $20 \mathrm{psia}$, find the temperature at the exit, in ${ }^{\circ} \mathrm{F}$. 3 sig figs

\section{Answer:}

\section{CHECK}

$\mathrm{R} 134 \mathrm{a}$ enters an ideal, adiabatic compressor as saturated vapor at $120 \mathrm{kPa}$. If it exits at a temperature of $60^{\circ} \mathrm{C}$, what is the pressure at the exit, in $\mathrm{MPa}$ ? 3 sig figs

\section{Answer:}

\section{CHECK}

Steam enters a reversible, adiabatic nozzle at negligible velocity, a pressure of $500 \mathrm{kPa}$, and a temperature of $400^{\circ} \mathrm{C}$. It exits at $100 \mathrm{kPa}$. Find the exit velocity, in $\mathrm{m} / \mathrm{s}$. $3 \mathrm{sig}$ figs

\section{Answer:}

\section{CHECK}




\section{Day 2: Worksheet problem before the first quizlet}

1. An insulated piston/cylinder filled with oxygen goes through a reversible expansion process. If the initial temperature is $60^{\circ} \mathrm{F}$, the initial pressure is $50 \mathrm{psia}$, and the final pressure is $20 \mathrm{psia}$, find the final temperature.

a. Using variable specific heats (Ans: $400.6 \mathrm{R}$ )

b. Using constant specific heats (Ans: $400.6 \mathrm{R}$ )

\section{Day 2: Three of the ten problems in Quizlet 1}

A large slab of concrete, $5 \mathrm{~m} \times 8 \mathrm{~m} \times 0.3 \mathrm{~m}$, is used as a thermal storage mass in a solar-heated home. If the slab cools overnight from $23^{\circ} \mathrm{C}$ to $18^{\circ} \mathrm{C}$, what is the entropy change associated with this process, in $\mathrm{kJ} / \mathrm{K}$ ? Use 3 sig. figs.

Answer:

CHECK

Find $\mathrm{s}_{2}-\mathrm{s}_{1}$ in $\mathrm{kJ} / \mathrm{kgK}$ using constant specific heats. Substance: air. $P_{1}=200 \mathrm{kPa}, T_{1}=7^{\circ} \mathrm{C} . P_{2}=100$ $\mathrm{kPa}, \mathrm{T}_{2}=347^{\circ} \mathrm{C}$. Use 3 sig. figs.

Answer:

\section{CHECK}

Find $\mathrm{s}_{2}-\mathrm{s}_{1}$ in $\mathrm{kJ} / \mathrm{kgK}$ using variable specific heats. Substance: hydrogen. $\mathrm{P}_{1}=100 \mathrm{kPa}, \mathrm{T}_{1}=727^{\circ} \mathrm{C} . \mathrm{P}_{2}$ $=300 \mathrm{kPa}, \mathrm{T}_{2}=25^{\circ} \mathrm{C}$. Use 3 sig. figs.

Answer:

CHECK

\section{Day 2: Worksheet problem before the second quizlet}

2. Air enters an insulated turbine operating at steady state at $650 \mathrm{kPa}, 687^{\circ} \mathrm{C}$ and exits at $100 \mathrm{kPa}$, $327^{\circ} \mathrm{C}$. Determine the work developed in $\mathrm{kJ} / \mathrm{kg}$ of air flowing and whether the expansion is internally reversible, irreversible, or impossible. (Ans: $393.5 \mathrm{~kJ} / \mathrm{kg},+0.0249 \rightarrow$ irreversible (real)) 


\section{Day 3: Three of the five problems in the first quizlet}

Air enters an insulated, reversible turbine at $1040 \mathrm{~K}, 277.7 \mathrm{kPa}$ and exits at $120 \mathrm{kPa}$. Find the enthalpy at the exit in $\mathrm{kJ} / \mathrm{kg}$ using variable specific heats. $3 \mathrm{sig}$. figs.

\section{Answer:}

\section{CHECK}

The maximum temperature in an ideal Otto cycle is $2490^{\circ} \mathrm{F}$ and its compression ratio is 7 . Find the temperature at the end of the power stroke in ${ }^{\circ} \mathrm{F}$ using an air-standard analysis. $3 \mathrm{sig}$. figs.

Answer:

CHECK

$0.1 \mathrm{~kg}$ of air in a piston/cylinder in a car engine undergoes an isentropic compression stroke. If the air starts at $295 \mathrm{~K}, 100 \mathrm{kPa}$ and the engine has a compression ratio of 6.5 , find the work for the compression stroke in $\mathrm{kJ}$. Use a cold-air standard analysis. 3 sig. figs.

\section{Answer:}

\section{CHECK}

\section{Day 3 Otto Cycle problems. (Each part is a quizlet problem).}

Quizlet 2: Air-Standard Otto Cycle:

1. An ideal air-standard Otto cycle has a compression ratio of 9. At the beginning of compression, $P_{1}=100 \mathrm{kPa}$ and $T_{1}=300 \mathrm{~K}$. The heat addition per unit mass of air is 1350 $\mathrm{kJ} / \mathrm{kg}$.

Draw a T-s diagram of the cycle:

Use 3 sig figs for all:

a) Find the specific internal energy at the end of the compression process in $\mathrm{kJ} / \mathrm{kg}$

b) Find the specific internal energy at the beginning of the power stroke in $\mathrm{kJ} / \mathrm{kg}$

c) Find the specific internal energy at the end of the power stroke in $\mathrm{kJ} / \mathrm{kg}$

d) Find the specific work for the compression stroke in $\mathrm{kJ} / \mathrm{kg}$

e) Find the net work for the cycle in $\mathrm{kJ} / \mathrm{kg}$ 
f) Find the thermal efficiency of the cycle in percent.

g) Find the maximum temperature in the cycle, in Kelvin

Quizlet 3: Cold-Air-Standard Otto Cycle: (3 sig. figs.)

2. Using the same cycle as in number 1 , use cold-air standard analysis to

a) Find the thermal efficiency of the cycle in percent

b) Find the temperature at the end of the compression process, in Kelvin

c) Find the maximum temperature, in Kelvin

d) Find the temperature at the end of the power stroke

e) Find the net work for the cycle in $\mathrm{kJ} / \mathrm{kg}$ (Note: You can do this without finding work for the separate processes.) 